Sir,

About bariatric surgery and diabetic retinopathy; the debate continues

We read with interest the paper by Chen et al, ${ }^{1}$ based on a retrospective study of 102 diabetic patients with no retinopathy $(68 \%)$ or pre-existing retinopathy $(32 \%)$ reevaluated up to 4 years after bariatric surgery. Chen et al ${ }^{1}$ found that retinopathy appeared or progressed in $24 \%$ of patients, while retinopathy of any degree remained stable in $72 \%$ of patients and improved in $4 \%$ of patients. Young age, male gender, high pre-operative $\mathrm{HbA} 1 \mathrm{c}$, and presence of pre-operative retinopathy were the significant predictors of worsening postoperatively.

Chen et $a l^{1}$ conclude that bariatric surgery does not prevent progression of DR, and that future prospective studies with long follow-up are required to clarify the duration of risk.

Similar results, but also contrasting results, have been reported in the literature, leading to opposite interpretations. ${ }^{2,3}$ One should consider first that all these findings came from uncontrolled studies, and therefore one might hypothesize that similar or even worse results might apply for diabetic patients receiving only medical treatment instead of bariatric surgery; it is known that rapid improvement in glycaemic control may cause a paradoxical worsening in DR. ${ }^{1}$ Second, in a large cohort study on about 4600 diabetic patients undergoing bariatric surgery, patients who experienced type 2 diabetes remission had 29\% lower risk of incident microvascular disease compared with patients who never remitted. Among patients who experienced a relapse after remission, the length of time spent in remission was inversely related to the risk of incident microvascular disease. ${ }^{4}$

Nevertheless, these data suggest that bariatric surgery, if any, can prevent appearance of retinopathy, but is unable to prevent deterioration of pre-existing retinopathy, as outlined in a meta-analysis that took into consideration only controlled studies. ${ }^{5}$

\section{Conflict of interest}

The authors declare no conflict of interest.

\section{References}

1 Chen Y, Laybourne JP, Sandinha MT, de Alwis NM, Avery P, Steel $\mathrm{DH}$ et al. Does bariatric surgery prevent progression of diabetic retinopathy? Eye 2017; 31: 1131-1139.

2 Cheung D, Switzer NJ, Ehmann D, Rudnisky C, Shi X, Karmali S. The impact of bariatric surgery on diabetic retinopathy: a systematic review and meta-analysis. Obes Surg 2015; 25: 1604-1609.

3 Pontiroli AE, Ceriani V. Does bariatric surgery influence diabetic retinopathy? Obes Surg 2015; 25: 1066-1067.

4 Coleman KJ, Haneuse S, Johnson E, Bogart A, Fisher D, O'Connor PJ et al. Long-term microvascular disease outcomes in patients with type 2 diabetes after bariatric surgery: evidence for the legacy effect of surgery. Diabetes Care 2016; 39: 1400-1407.

5 Merlotti C, Ceriani V, Morabito A, Pontiroli AE. Bariatric surgery and diabetic retinopathy: a systematic review and meta-analysis of controlled clinical studies. Obes Rev 2017; 18 : 309-316.

\section{AE Pontiroli ${ }^{1}$, C Merlotti $^{2}$, V Ceriani ${ }^{3}$ and A Morabito ${ }^{1}$}

${ }^{1}$ Dipartimento di Scienze della Salute, Università degli Studi di Milano, Milan, Italy

${ }^{2}$ Dipartimento di Medicina, Ospedale San Paolo, Milan, Italy

${ }^{3}$ Dipartimento di Chirurgia, Istituto Multimedica, Milan, Italy

E-mail: antonio.pontiroli@unimi.it

Eye (2018) 32, 652; doi:10.1038/eye.2017.217; published online 13 October 2017

Sir,

Response to 'About bariatric surgery and diabetic retinopathy; the debate continues'

We thank Pontiroli et al for their response to our article. As acknowledged in our article, a control group would have strengthened our study; however, a well-balanced or randomised design is difficult to achieve in bariatric surgery. Due to this limitation, we did not attempt to conclude a causal relationship between bariatric surgery and progression in diabetic retinopathy (DR). On the basis of our observations, we highlighted that contrary to common assumption, patients can continue to experience progression in their DR post-bariatric surgery. ${ }^{1}$ Therefore, it is of utmost importance that both patients and healthcare providers are aware of this potential risk and ensure patients attend regular DR screening.

We read with interest the recent cohort study that showed reduced incidence of microvascular complications in diabetic patients who experienced remission post-bariatric surgery. ${ }^{2}$ This is supportive of our findings, which showed that worsening of DR over time was more common in patients in whom glycaemic control failed to improve significantly postoperatively. ${ }^{1}$ Unfortunately, direct comparison with our study result is restricted by a number of factors-Coleman et al used ICD-9 diagnosis codes to define DR, which differs from the NSC grading system; detection of DR was dependent on patients seeking care rather than screening on all participants, and therefore there is a risk of misclassification as patients can experience asymptomatic deterioration in DR; follow-up was limited to 13 months compared to 4 years in our study. As highlighted by Mertlotti et $a l^{3}$ in their recent meta-analysis, based on available data from controlled studies-bariatric surgery may prevent new incident cases of DR, but currently available studies are not sufficient to support any systematic effect on progression or regression of DR. A number of limitations were noted-controlled studies were few, there was a low incidence of new cases of retinopathy and follow-up period was in general short. Therefore, as emphasised by them and many similar publications, further study is required to draw firm conclusions on the effect of bariatric surgery on DR, but progression of retinopathy can clearly still occur despite, in some cases, normalisation of glucose levels. 


\section{Conflict of interest}

The authors declare no conflict of interest.

\section{References}

1 Chen Y, Laybourne JP, Sandinha MT, de Alwis NM, Avery P, Steel DH. Medscape. Does bariatric surgery prevent progression of diabetic retinopathy? Eye 2017; 31: 1131-1139.

2 Coleman KJ, Haneuse S, Johnson E, Bogart A, Fisher D, O'Connor PJ et al. Long-term microvascular disease outcomes in patients with type 2 diabetes after bariatric surgery: evidence for the legacy effect of surgery. Diabetes Care 2016; 39: 1400-1407.

3 Merlotti C, Ceriani V, Morabito A, Pontiroli AE. Bariatric surgery and diabetic retinopathy: a systematic review and meta-analysis of controlled clinical studies. Obes Rev 2017; 18: 309-316.

Y Chen ${ }^{1}$, JP Laybourne1, MT Sandinha1, NMW de Alwis², $\mathrm{P}$ Avery $^{3}$ and DH Steel ${ }^{1,4}$

${ }^{1}$ Sunderland Eye Infirmary, Queen Alexandra Road, Sunderland, UK

${ }^{2}$ Department of Diabetes and Endocrinology, Sunderland Royal Hospital, Kayll Road, Sunderland, UK

${ }^{3}$ School of Mathematics \& Statistics, Newcastle

University, Newcastle Upon Tyne, UK
${ }^{4}$ Institutes of Genetic Medicine, Newcastle University, Newcastle Upon Tyne, UK

E-mail: chen_yunzi@hotmail.com

Eye (2018) 32, 652-653; doi:10.1038/eye.2017.219;

published online 20 October 2017

Sir,

Research attitudes and perceived barriers to conducting research among ophthalmology trainees

High-quality research forms the basis of evidence-based medicine. A strong grasp of the fundamental principles of research is essential for all doctors of all grades. Studies have shown that research training embedded within clinical training programmes helps to promote trainees' critical thinking and analysis, improve quality of patient care and facilitate post-training academic output. ${ }^{1}$

Unlike other surgical trainees, participation in research activities is not mandated-albeit highly recommendedwithin the ophthalmology training programmes in the UK. However research participation is often deterred by the lack of time and inadequate research skills. ${ }^{2,3}$ In 2017 we sent out a 26-item questionnaire-based survey to all the ophthalmology trainees in the North East of England to examine their research attitudes and perceived barriers to conducting research. Trainees were asked to rank the utility of RSTA — a dedicated session allocated for

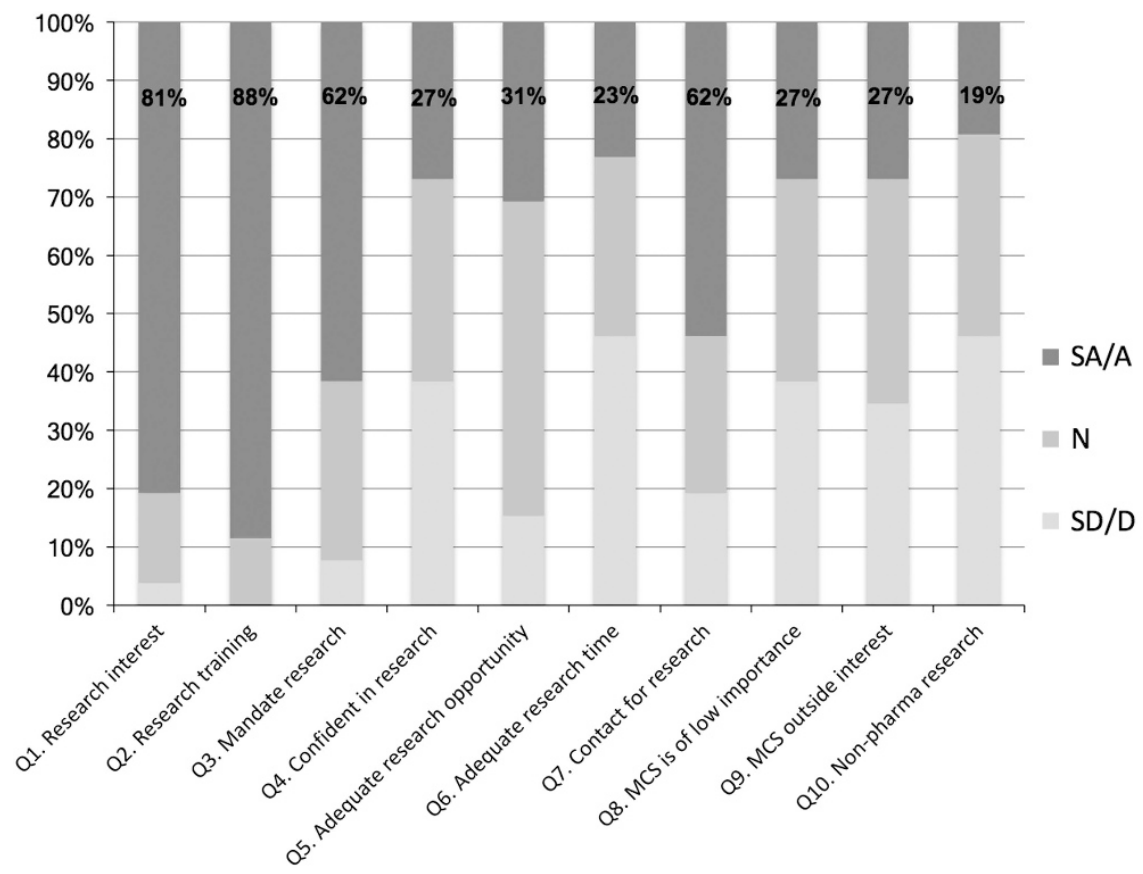

Figure 1 The summary of research attitudes of 26 ophthalmology trainees in the North East of England. SA-Strongly agree; A-Agree; N-Neutral; D—Disagree; SD—Strongly disagree; MCS-Multicentre study. Q1: I am interested in research. Q2: Research training is important for the trainees. Q3: Research should be mandated as part of the clinical training. Q4: I am confident in conducting research. Q5: I have been given enough opportunity to participate in research. Q6: I feel that I have enough time to participate in research. Q7: I know whom to speak to if I want to participate in research. Q8: I feel that participation in multicentre studies is of low importance because I am not included as a named co-author. Q9: I am interested in participating in multicentre studies but the research subject is not within my interest. Q10: I am only interested in non-pharmaceutical led research. 